\title{
Ayuuk-Spanish Neural Machine Translator
}

\author{
Delfino Zacarías \\ Facultad de Estudios Superiores Acatlán, \\ Universidad Nacional Autónoma de México \\ delfino.zacariasecomunidad.unam.mx

\begin{abstract}
Ivan Meza
Instituto de Investigaciones en Matemáticas Aplicadas y en Sistemas, Universidad Nacional Autónoma de México

ivanvladimireturing.iimas.unam.mx
\end{abstract}

\begin{abstract}
This paper presents the first neural machine translator system for the Ayuuk language. In our experiments we translate from Ayuuk to Spanish, and from Spanish to Ayuuk. Ayuuk is a language spoken in the Oaxaca state of Mexico by the Ayuukjä'äy people (in Spanish commonly known as Mixes). We use different sources to create a low-resource parallel corpus, more than 6,000 phrases. For some of these resources we rely on automatic alignment. The proposed system is based on the Transformer neural architecture and it uses sub-word level tokenization as the input. We show the current performance given the resources we have collected for the San Juan Güichicovi variant, they are promising, up to 5 BLEU. We based our development on the Masakhane project for African languages.
\end{abstract}

\section{Introduction}

In recent years the efforts to preserve and promote the creation of NLP tools for the native languages of the Americas have increased, particularly addressing the challenges that this endeavour requires (Mager et al., 2018). Machine Translation (MT) has become one of the main goals to pursue since in the long term it might offer benefits to the communities that speak such languages. For instance, it might provide access to knowledge in their native language and facilitate access to services such legal, medical and finance assistance. In this work, we explore this avenue for the San Juan Güichicovi variant of the Ayuuk language, mainly because one of the authors is a native speaker of this variant. To our knowledge there has not been a construction of such a system for the Ayuuk although other variants ${ }^{1}$ are available in the JW300 Corpus (Agić and Vulić, 2019).

In this work we rely in multiple previous work. At the core of our proposal we follow the steps from

\footnotetext{
${ }^{1}$ Coatlán Mixe (ISO 639-3 mco), Ayuuk of the Coatlán region.
}

the Masakhane project ${ }^{2}$ which focuses on African Languages (Nekoto et al., 2020). We also rely on the following libraries:

- For the automatic alignment of our resources we use the YASA alignment (Lamraoui and Langlais) $)^{3}$

- For the tokenization we use subword-nmt library $^{4}$ (Sennrich et al., 2016)

- For the training of our models we use JoeyNMT ${ }^{5}$ (Kreutzer et al., 2019).

With these tools we developed our code base that can be consulted online together with the part of the corpus which is freely available ${ }^{6}$.

\section{Ayuuk from San Juan Güichicovi}

Ayuukjä'äy can be translated as people of the mountains, most them can be located in 24 municipalities of the Oaxaca state. They are the native speakers of the Ayuuk language with approximately 139,760 speakers in Mexico. The Ayuuk language, which has an ISO 639-3 code mir, belongs to the mixezoqueana linguistic family. This linguistic family is composed by the Mixe and Zoque subfamilies ${ }^{7}$. In particular, the Mixe subfamily also includes Mixe of Oaxaca, Sayula Popoluca and Oluta Popoluca languages. For Ayuuk there are six main variants of the language, among these the Mixe bajo to which the San Juan Güichicovi variant belongs to. At

\footnotetext{
${ }^{2}$ https: // www . masakhane.io/ (last visited march 2021)

${ }^{3}$ https://github.com/anoidgit/yasa (last visited march 2021)

${ }^{4}$ https://github.com/rsennrich/ subword-nmt (last visited march 2021).

${ }^{5}$ https: //github.com/joeynmt/joeynmt (last visited march 2021)

${ }^{6}$ https://github.com/DelfinoAyuuk/ corpora_ayuuk-spanish_nmt

${ }^{7}$ For further information visit about the mixe-zoqueana family https://glottolog.org/resource/ languoid/id/mixe1284
} 
this municipality it can be estimated there is approximately 18,298 speakers of the variant. It is important to notice that it is estimated that only 3,205 are monolinguist.

The San Juan Güichicovi's Ayuuk variant does not has a normalized orthography, there are efforts to agree on orthographic conventions however there are strong positions related to number of consonants. One of these positions, it is known as the "bodegeros" position which proposes 20 consonants (see 1b.a) (Willett et al., 2018) vs "petakeros" which proposes a reduction to 13 (see 1b.b) (Reyes Gómez, 2005). In terms of vowels, this variant has six (see 2) which contrast with the other variants of Ayuuk which can have up to nine vowels.

(1) a. b ch d ds g j kl m n ñ prst ts w x y'

b. ptkx ts m n wy j $1 \mathrm{rs}$,

(2) a e ë i o u

The following are examples of San Juan Güichicovi'sAyuuk these were taken from short stories recollected and written by Albino Pedro Juan a native speaker and preserver of the language.

(3) Jantim xyondaak ja koy jadu'un.

The bunny become happy.

El conejo se puso feliz.

(4) Kabëk je'e ti y'ok ëjy y'ok nójnë. When everything become silence.

Cuando todo se silencia.

\subsection{Spanish}

In the case of Spanish, our system produces translations in Mexican Spanish which belongs to the American Spanish variant ${ }^{8}$, we identify the language by the es ISO-639-1 code.

\section{The parallel corpus}

For the creation of the parallel corpus we collected samples from different sources for which there was a available translation between Ayuuk and Spanish, see Table 1.

Since we have a diverse source of linguistic sources it was necessary to normalize the orthography. For this we follow the proposal from SagiVela González (2019) who has followed the unification of the Ayuuk language avoiding taking sides on the controversy about the number of consonants.

\footnotetext{
${ }^{8}$ https://glottolog.org/resource/ languoid/id/amer1254 (visited, last visited march 2021)
}

\begin{tabular}{|c|c|c|}
\hline Resource & es & mir \\
\hline The bible & Open & No open \\
\hline Songs and poems & No open & No open \\
\hline The Mexican constitution & Open & No open \\
\hline $\begin{array}{l}\text { Personal colection of } \\
\text { Albino Pedro Juan }\end{array}$ & No open & No open \\
\hline Esopo Fables & Open & No open \\
\hline $\begin{array}{l}\text { National archive of } \\
\text { indigenous languages }\end{array}$ & No open & Open \\
\hline Social network $^{\mathrm{a}}$ & Open & Open \\
\hline The dragon and the rabbit ${ }^{\mathrm{a}}$ & Open & Open $^{b}$ \\
\hline Phrases translated by author ${ }^{\mathrm{a}}$ & Open $^{c}$ & Open \\
\hline
\end{tabular}

Table 1: Source of data collected

Mainly we made two replacements: $\tilde{n} / n y$ and $c h / t s y$ Some of the works were already aligned, others not. For those not aligned we created automatic alignments using the YASA tool (Lamraoui and Langlais). We discarded all empty and double alignments. Normalization and automatic alignments were manually verified by one of the authors. The corpus keep differences among both normalization variants: petakeros and bodegeros.

Finally, we randomly split the sentences into training, development and testing sets. For our experimentation we created two split versions, one strict and one random. In the strict version we use all the phrases from the National archive of indigenous languages (Lyon, 1980) as a test. Since these sentences are linguistically motivated and aim to show linguistic aspects of the language they tend to be harder to translate; This split resulted in 5, 847/700/912 (train/dev/test). In the random split we randomly sample sentences from our sources, the final split resulted in 5,941/700/912 (train/dev/test). Notice that amount of phrases among splits changes, this is because after separating the test phrases, we remove repeated or similar phrases for the train/dev sets. Our intuition was to have a more uniform training/validation for the random split while the test follows the distribution of the original sources. We mimic this procedure for the strict sample.

\section{Neural Architecture}

Our translation model is based on the Transformer architecture (Vaswani et al., 2017). We use an encoder-decoder setting. For our experiments we 

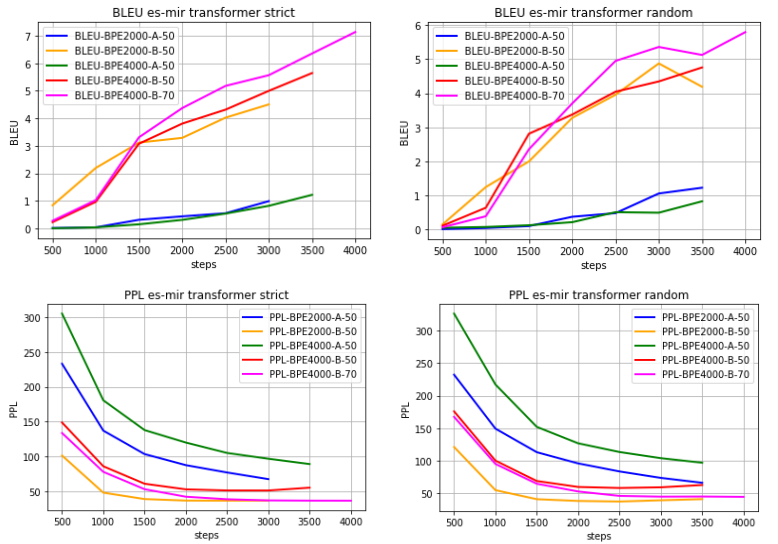

Figure 1: Perplexity and BLEU of es-mir in development set.

have two configurations for both encoder and decoder:

A Number of layers: 3, number of heads: 4, Input embedding dimensionality: 64 , embedding dimensionality: 64, batch size: 128 .

B Number of layers: 6, number of heads: 4, input embedding dimensionality: 256 , embedding dimensionality: 256, batch size: 128 .

These models were trained in a server with two Tesla V100 GPUs. To obtain a model it usually take us around $2 h$ for a 100 epochs. We also were able to reproduce the experiments in the Colaboratory platform.

\section{Experiments and results}

As described in the previous section we have two different versions of our splits, strict and random. Per split we performed five experiments, two for configuration with fewer layers $(A)$, and three for the configuration with more layers $(B)$. We also modified: $a$ ) the maximum length of the phrase (50 or 70) $b$ ) the vocabulary of the BPE sub-word algorithm (we tested 2000 or 4000). Figure 1 shows the perplexity and the BLEU score in the development set during training for the direction Spanish (es) to Ayuuk (mir). The first part of the Table 2, columns two to five, presents the results on the development and test sets.

Figure 2 shows the lerning curve on the direction of translation Ayuuk (mir) to Spanish (es). The second part of the table 2, columns six to nine, presents the results on the development and test for this translation direction.
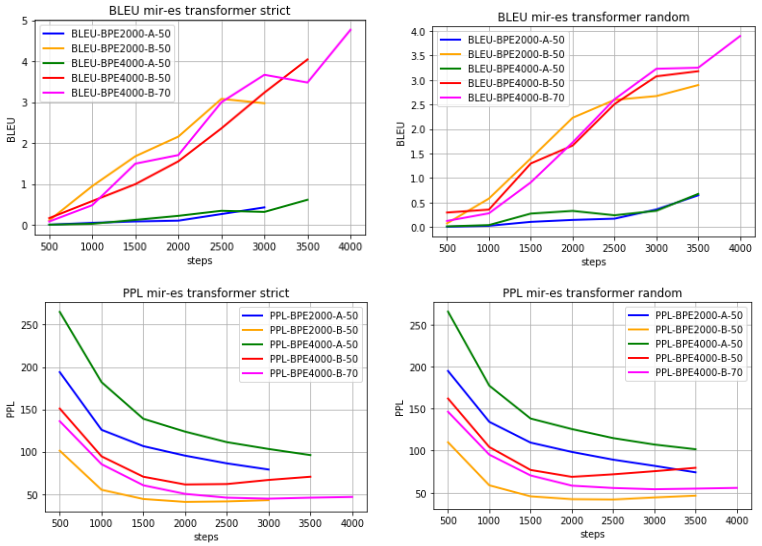

Figure 2: Perplexity and BLEU of mir-es in development set.
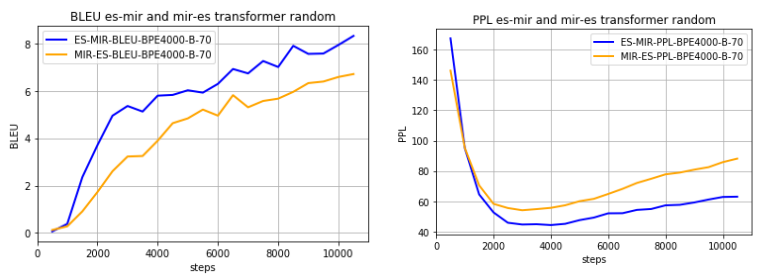

Figure 3: Perplexity and BLEU of es-mir and mir-es training with 250 epochs.

As we can appreciate these sets of experiments show that the translation is possible. We have some gains on the model with more layers $(B)$, this is not trivial since we have a small amount of training data. On the other hand, the strict split as expected shows to be very difficult to translate, the BLEU scores are minimal. However with the random splits the BLEU scores are more promising. We also observe there that in the current setting it is more "easy" to translate from Spanish to Ayuuk than the other direction. Finally, we perform a larger experimentation with 250 epochs using the $B$ configuration, following the intuition we haven reach the right performance with 100 . Figure 3 shows the learning curve on the development set, the bottom part of Table 2 shows our final results using the random split.

\section{Conclusions and Further work}

Previous experiences on MT based on deep learning architecture, particularly on seq2seq settings, for native languages of the Americas have not been promising (Mager and Meza, 2018). In particular, because there is little to none training data. However, our work shows that a standard model based on the Transformer architecture and under 


\begin{tabular}{|c|c|c|c|c|c|c|c|c|}
\hline Configuration A 100 epochs & \multicolumn{2}{|c|}{ Strict es-mir } & \multicolumn{2}{|c|}{ Random es-mir } & \multicolumn{2}{|c|}{ Strict mir-es } & \multicolumn{2}{|c|}{ Random mir-es } \\
\hline 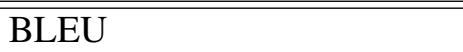 & $\overline{c \text { dev }}$ & "test & 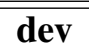 & "test & 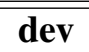 & "test & 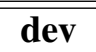 & 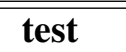 \\
\hline $\begin{array}{l}\text { Max lenght } 50 \\
\text { BPE } 2000\end{array}$ & 1.72 & 0.05 & 1.66 & 1.71 & 0.64 & 0.10 & 0.91 & 0.66 \\
\hline $\begin{array}{l}\text { Max lenght } 50 \\
\text { BPE } 4000\end{array}$ & 2.03 & 0.10 & 1.21 & 1.24 & 1.02 & 0.16 & 0.93 & 0.83 \\
\hline Configuration B 100 epochs & \multicolumn{2}{|c|}{ Strict es-mir } & \multicolumn{2}{|c|}{ Random es-mir } & \multicolumn{2}{|c|}{ Strict mir-es } & \multicolumn{2}{|c|}{ Random mir-es } \\
\hline 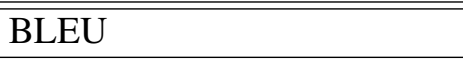 & 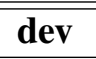 & test & 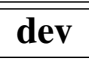 & test & 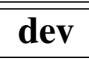 & test & $\overline{\mathrm{dev}}$ & test \\
\hline $\begin{array}{l}\text { Max lenght } 50 \\
\text { BPE } 2000\end{array}$ & 3.91 & 0.10 & 3.59 & 3.70 & 2.21 & 0.41 & 2.49 & 2.72 \\
\hline $\begin{array}{l}\text { Max lenght } 50 \\
\text { BPE } 4000\end{array}$ & 5.02 & 0.13 & 4.17 & 4.20 & 2.33 & 0.28 & 2.13 & 2.23 \\
\hline $\begin{array}{l}\text { Max lenght } 70 \\
\text { BPE } 4000\end{array}$ & 7.58 & 0.10 & 5.83 & 5.56 & 4.03 & 0.27 & 3.64 & 3.52 \\
\hline Configuration B 250 epochs & \multicolumn{4}{|c|}{ Random es-mir } & \multicolumn{4}{|c|}{ Random mir-es } \\
\hline 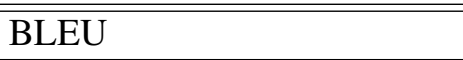 & \multicolumn{2}{|c|}{ dev } & \multicolumn{2}{|c|}{ test } & \multicolumn{2}{|c|}{$\overline{\mathrm{dev}}$} & \multicolumn{2}{|c|}{ test } \\
\hline $\begin{array}{l}\text { Max lenght } 70 \\
\text { BPE } 4000\end{array}$ & \multicolumn{2}{|c|}{5.83} & \multicolumn{2}{|c|}{5.56} & \multicolumn{2}{|c|}{3.64} & \multicolumn{2}{|c|}{3.52} \\
\hline
\end{tabular}

Table 2: BLEU scores of es-mir and mir-es.

extremely low resource setting can produce some results. They are still low for normal standards of the MT field however they are promising for the future.

In order to improve the performance of the system future work will focus on:

1. Collecting more data, paying attention to other variants of the Ayuuk language.

2. Although the strict setting strongly penalizes the evaluation, we will continue using linguistic motivated phrases as a good bar to evaluate our progress.

3. At this moment we rely on sub-word of the phrases, however our approach could benefit from a deeper morphology analysis (Kann et al., 2018).

4. Our normalization will continue respecting the petakeros and bodegeros positions, and for other variants we also incorporate positions regarding the number of vowels.

\section{Acknowledgements}

The authors thank CONACYT for the computer resources provided through the INAOE Supercomputing Laboratory's Deep Learning Platform for Language Technologies. We also thank the project
"Traducción automática para lenguas indígenas de México" PAPIIT-IA104420, UNAM.

\section{References}

Željko Agić and Ivan Vulić. 2019. JW300: A widecoverage parallel corpus for low-resource languages. In Proceedings of the 57th Annual Meeting of the Association for Computational Linguistics, pages 3204-3210, Florence, Italy. Association for Computational Linguistics.

Katharina Kann, Jesus Manuel Mager Hois, Ivan Vladimir Meza-Ruiz, and Hinrich Schütze. 2018. Fortification of neural morphological segmentation models for polysynthetic minimal-resource languages. In Proceedings of the 2018 Conference of the North American Chapter of the Association for Computational Linguistics: Human Language Technologies, Volume 1 (Long Papers), pages 47-57, New Orleans, Louisiana. Association for Computational Linguistics.

Julia Kreutzer, Jasmijn Bastings, and Stefan Riezler. 2019. Joey NMT: A minimalist NMT toolkit for novices. In Proceedings of the 2019 Conference on Empirical Methods in Natural Language Processing and the 9th International Joint Conference on Natural Language Processing (EMNLP-IJCNLP): System Demonstrations, pages 109-114, Hong Kong, China. Association for Computational Linguistics.

Fethi Lamraoui and Philippe Langlais. Yet another fast, robust and open source sentence aligner. time to reconsider sentence alignment. XIV Machine Translation Summit. 
Don D. Lyon. 1980. Mixe de Tlahuitoltepec, Oaxaca, Archivo de Lenguas Indígenas de México. Colegio de México, México.

Manuel Mager, Ximena Gutierrez-Vasques, Gerardo Sierra, and Ivan Meza-Ruiz. 2018. Challenges of language technologies for the indigenous languages of the Americas. In Proceedings of the 27th International Conference on Computational Linguistics, pages 55-69, Santa Fe, New Mexico, USA. Association for Computational Linguistics.

Manuel Mager and Ivan Meza. 2018. Hacia la traducción automática de las lenguas indigenas de méxico. Proceedings of the $\mathrm{DH}$.

Wilhelmina Nekoto, Vukosi Marivate, Tshinondiwa Matsila, Timi Fasubaa, Taiwo Fagbohungbe, Solomon Oluwole Akinola, Shamsuddeen Muhammad, Salomon Kabongo Kabenamualu, Salomey Osei, Freshia Sackey, Rubungo Andre Niyongabo, Ricky Macharm, Perez Ogayo, Orevaoghene Ahia, Musie Meressa Berhe, Mofetoluwa Adeyemi, Masabata Mokgesi-Selinga, Lawrence Okegbemi, Laura Martinus, Kolawole Tajudeen, Kevin Degila, Kelechi Ogueji, Kathleen Siminyu, Julia Kreutzer, Jason Webster, Jamiil Toure Ali, Jade Abbott, Iroro Orife, Ignatius Ezeani, Idris Abdulkadir Dangana, Herman Kamper, Hady Elsahar, Goodness Duru, Ghollah Kioko, Murhabazi Espoir, Elan van Biljon, Daniel Whitenack, Christopher Onyefuluchi, Chris Chinenye Emezue, Bonaventure F. P. Dossou, Blessing Sibanda, Blessing Bassey, Ayodele Olabiyi, Arshath Ramkilowan, Alp Öktem, Adewale Akinfaderin, and Abdallah Bashir. 2020 Participatory research for low-resourced machine translation: A case study in African languages. In Findings of the Association for Computational Linguistics: EMNLP 2020, pages 2144-2160, Online. Association for Computational Linguistics.

J. Carlos Reyes Gómez. 2005. Aportes al proceso de enseñanza aprendizaje de la lectura y la escritura de la lengua ayuuk. Centro de Estudios Ayuuk-Universidad Indígena Intercultural Ayuuk, Oaxaca, México.

Ana Sagi-Vela González. 2019. El mixe escrito y el espejismo del buen alfabeto. Revista de Llengua $i$ Dret, (71).

Rico Sennrich, Barry Haddow, and Alexandra Birch. 2016. Neural machine translation of rare words with subword units. In Proceedings of the 54th Annual Meeting of the Association for Computational Linguistics (Volume 1: Long Papers), pages 17151725, Berlin, Germany. Association for Computational Linguistics.

Ashish Vaswani, Noam Shazeer, Niki Parmar, Jakob Uszkoreit, Llion Jones, Aidan N Gomez, Ł ukasz Kaiser, and Illia Polosukhin. 2017. Attention is all you need. In Advances in Neural Information Processing Systems, volume 30. Curran Associates, Inc.
Thomas Willett, Susan Graham, Valerie Hillman, Judith Williams, Miriam Becerra Bautista, Miriam Pérez Luría, Vivian Eberle-Cruz, Karina Araiza Riquer, Julia Dieterman, James Michael McCarty Jr, Victoriano Castañón López, and María Dolores Castañón Eugenio. 2018. Breve diccionario del mixe del Istmo Mogoñé Viejo, Oaxaca, primera edition. Instituto Linguistico de Verano, México, D.F. 